\title{
TURBULENT JETS ISSUING FROM RECTANGULAR NOZZLE WITH A RECTANGULAR NOTCH AT THE MIDSPAN
}

\begin{abstract}
Shigetaka FUJITA ${ }^{\bullet 1}$, Takashi HARIMA ${ }^{* 1}$ and Hideo OSAKA ${ }^{\bullet 2}$
Abstract: The turbulent flowfield of turbulent jet issuing from rectangular nozzle (Aspect Ratio=12.5) with a rectangular notch at the midspan, has been investigated experimentally. Four aspect ratios of rectangular notch (NAR: Notch Aspect Ratio) used in this experiment were 2.5, 7.5, 12.5 and 165. The Reynolds number based on the nozzle width $d$ and the exit mean velocity Ue, was kept constant 30000 (NAR=2.5 and 7.5), 15000 (NAR=12.5) and 13000 (NAR=165), respectively. Longitudinal mean velocity and turbulent intensities were measured using an X-array Hot-Wire Probe (5 $\mu \mathrm{m}$ in diameter, $1 \mathrm{~mm}$ effective length) operated by the linearized constant temperature anemometers (DANTEC), and the spanwise and the lateral mean velocities were measured using a yaw meter. The signals from the anemometers were passed through the low-pass filters and sampled using A.D. converter. The processing of the signals was made by a personal computer. Acquisition time of the signals was usually 80 seconds. From this experiment, it was revealed that the attachment of a rectangular notch to the rectangular jet suppressed the development of the turbulent velocity scales near the jet centre in the upstream region for the cases of NAR $\geq 7.5$.
\end{abstract}

\section{INTRODUCTION}

The rectangular jet is very useful in many cases of which the improvement of the entrainment rate or the promotion of mixing is necessary. However, in case of the employment of the rectangular jet to engineering fields, the elongation of the high velocity jet flow region is expected in many cases, for example, the uniformity plating technique and the air curtain (air shield and ventilating).

To these technical assignments, the extension of the potential core length and the suppression of the turbulent intensities seem to be effective methods. So far, Trentacoste and Sforza [1], and Sforza and Stasi [2] classified the flowfield of the rectangular jet into 3 regions (Potential Core region (PC region), Characteristic Decay region (CD region) and Axisymmetric Decay region (AD region)).

\footnotetext{
-1 Shigetaka FUJITA, Department of Mechanical and Electrical Engineering, Tokuyama College of Technology, Gakuendai, Shunan, Yamaguchi, 745-8585, Japan, fujita@tokuyama.ac.jp

-1 Takashi HARIMA, Department of Mechanical and Electrical Engineering, Tokuyama College of Technology, Gakuendai, Shunan, Yamaguchi, 745-8585, Japan, harima@tokuyama.ac.jp

-2 Hideo OSAKA, Department of Mechanical System, Hiroshima Institute of Technology, Miyake, Saekiku, Hiroshima, Hiroshima, 731-5193, Japan, h.ohsaka.xv@it-hiroshima.ac.jp
} 
Furthermore, Marsters and Fotheringham [3] clarified that the streamwise mean velocity profiles showed the saddle-back shape which was caused by the inward secondary flow, and the reduction rate of the streamwise velocity along the jet centreline showed the typical feature in the cases of $A R$ (Aspect Ratio) $\geq 6.44$. From the results mentioned above, it seems that the variation of $A R$ changes the PC length and eases the velocity reduction of $C D$ region for the rectangular jet. Furthermore, Fujita et al. reported that the PC length of both the finite cruciform jet [4] and the two-dimensional jet with a rectangular notch [5], showed larger values for $N A R$ (Notch Aspect Ratio) $=12.5$ because of the inward secondary flow on the two representative jet axes. Quinn and Marsters [6] also reported the promotion of PC length for the finite cruciform jet with finite axis length $(A R=11.97)$.

From these results, it was clarified that the attachment of a rectangular notch with $A R=10 \sim 15$ perpendicular to the rectangular jet promoted the magnitude of the inward secondary flow toward the jet centre region on the rectangular jet axis [7]. Furthermore, it was presumed that the attachment of a rectangular notch to the rectangular nozzle suppressed the turbulent intensities, significantly.

In this report, the turbulent flowfield of turbulent jet issuing from rectangular nozzle (Aspect Ratio=12.5) with a rectangular notch $(N A R=2.5,7.5,12.5$ and 165) at the midspan, has been investigated to clarify an effect of the attachment of a rectangular notch to the rectangular jet, experimentally.

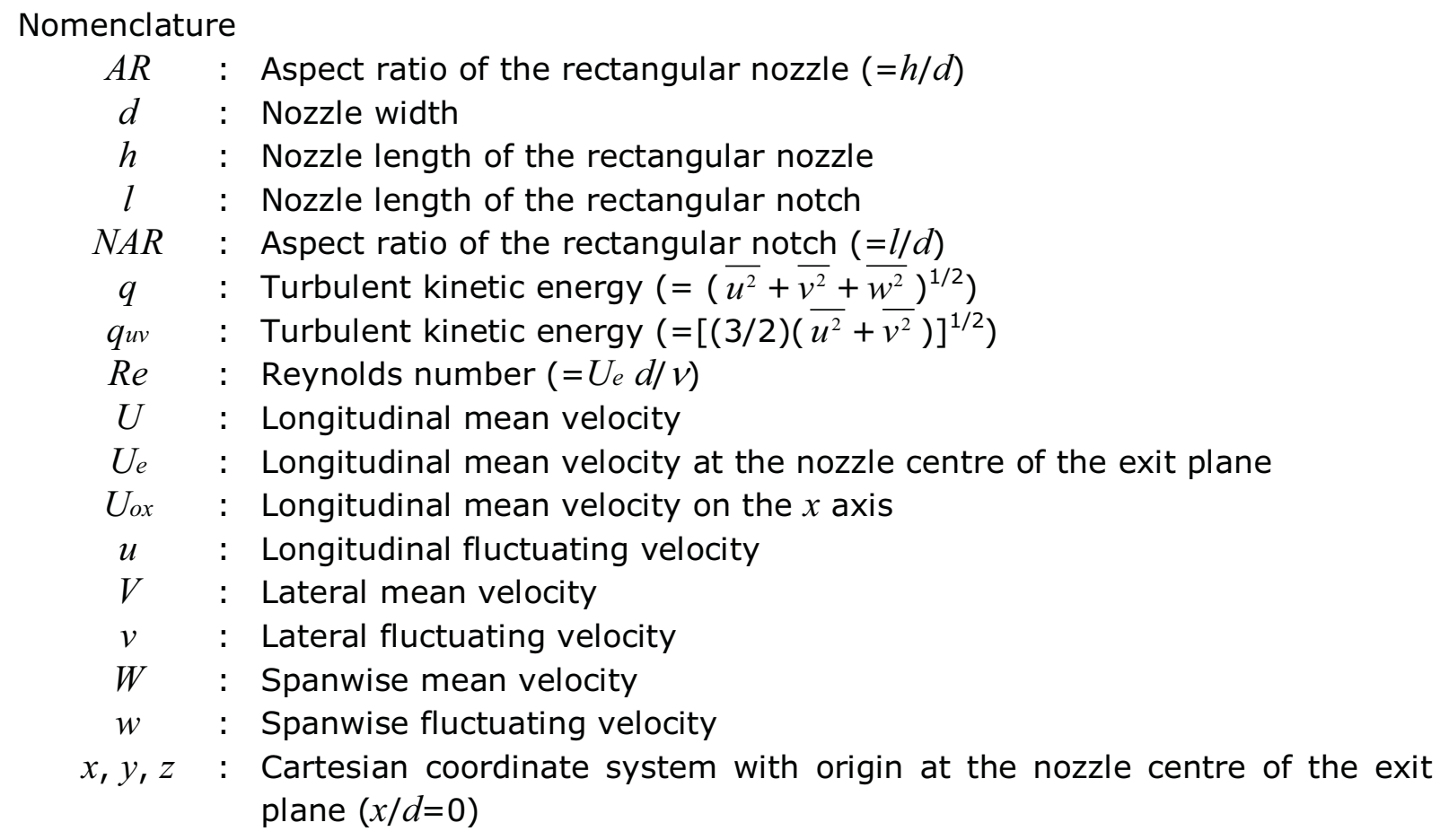

Greek Symbol

$v \quad:$ Kinetic viscosity of air

\section{Subscripts}

$e \quad:$ Value at the nozzle centre of the exit plane

ox : Value on the $x$ axis

rms : Root mean square 


\section{EXPERIMENTAL SETUP AND PROCEDURE}

The configuration of the flowfield and the coordinate system are presented in Figure 1. The rectangular nozzle with a rectangular notch is installed at the end of settling chamber. The jet flow facility consists of a turbo fan, a settling chamber fitted with honeycomb and mesh wire screens, and the rectangular nozzle with a rectangular notch. The main axis length $h$ and the width $d$ of the rectangular nozzle are $125 \mathrm{~mm}$ and $10 \mathrm{~mm}$ $(A R=12.5)$, respectively. On the other hand, the main axis length $l$ of the rectangular notch is $25 \mathrm{~mm}(N A R=2.5), 75 \mathrm{~mm}(N A R=7.5)$ and $125 \mathrm{~mm}(N A R=12.5)$ respectively, and each notch width $d$ is $10 \mathrm{~mm}$. For $N A R=165$, the rectangular notch length $l$ is 990 $\mathrm{mm}$ and the width $d$ is $6 \mathrm{~mm}$. Here, the value of $N A R$ for the rectangular nozzle is defined as zero, expediently.

Longitudinal mean velocity and turbulent intensities were measured using an X-array Hot-Wire Probe ( $5 \mu \mathrm{m}$ in diameter, $1 \mathrm{~mm}$ effective length) operated by the linearized constant temperature anemometers (DANTEC), and the spanwise and the lateral mean velocities were measured using a yaw meter. The signals from the anemometers were passed through the low-pass filters and sampled using A.D. converter. The processing of the signals was made by a personal computer. Acquisition time of the signals was usually 80 seconds. The exit plane Reynolds number based on the nozzle width $d$ and the exit mean velocity $U_{e}$, was kept constant 30000 (NAR=2.5 and 7.5), $15000(N A R=12.5)$ and $13000(N A R=165)$ throughout the present experiment, respectively. And the value of the nozzle exit turbulent intensity $u_{r m s} / U_{e}$ for all the $N A R$ cases were about $0.5 \times 10^{-2}$. In this experiment, the uncertainty associated with the longitudinal mean velocity $U$ is estimated at $\pm 3 \%$ of $U_{e}$, which includes calibration error of the linearized constant temperature anemometers, and that of the spanwise velocity $V$ and the lateral mean velocity $W$ at $\pm 6 \%$ of $U_{e}$, which includes calibration error of the yaw meter. Furthermore, the uncertainty is $\pm 5.6 \%$ of $U_{e}$ for the turbulent intensities.

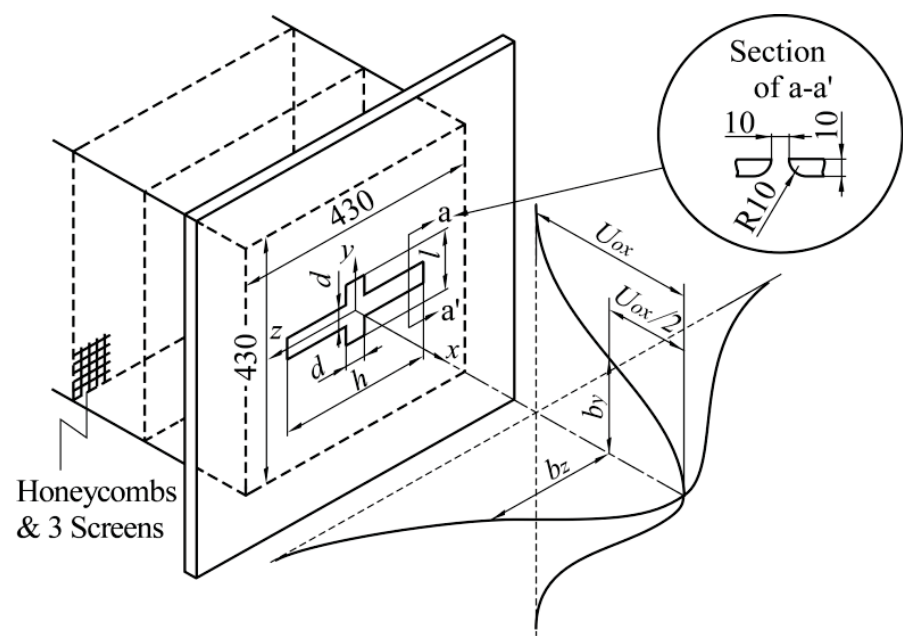

Figure 1: Configuration of the flowfield and the coordinate system 


\section{EXPERIMENTAL RESULTS AND DISCUSSION}

\subsection{CONFIGURATION OF THE MEAN VELOCITY FIELD}

At first, the mean velocity field will be shown for understanding the flowfield of the present jet. Figure 2 shows the streamwise variations of the longitudinal mean velocity

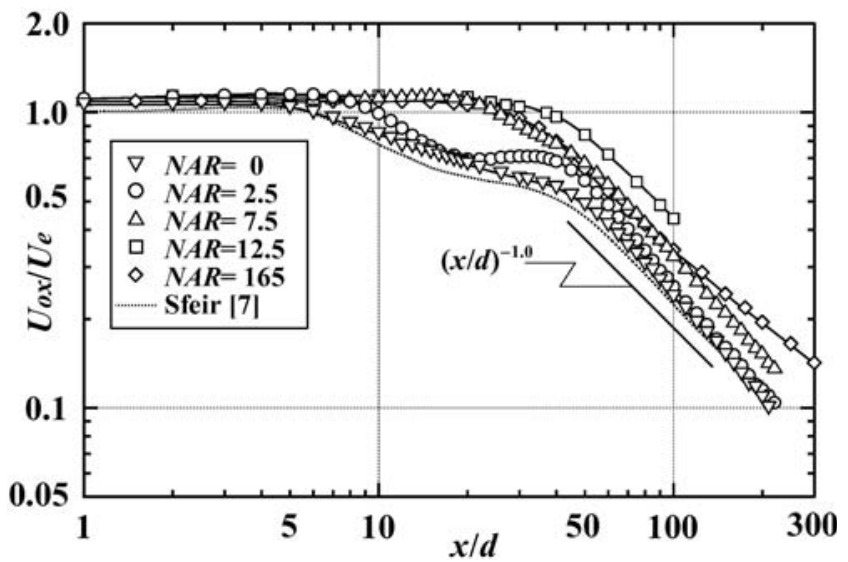

Figure 2: Longitudinal mean velocity profiles along the jet centreline
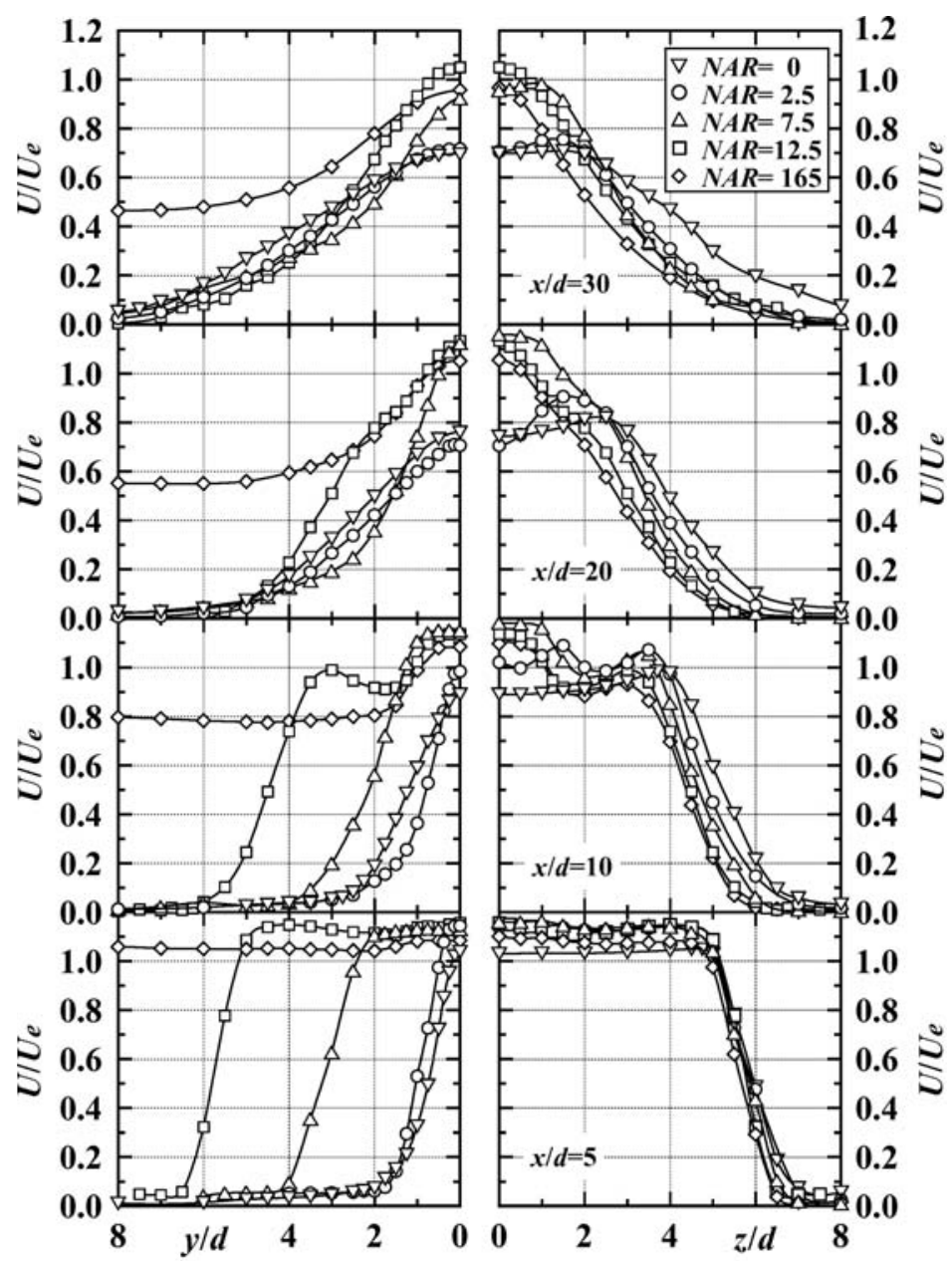

Figure 3: Longitudinal mean velocity profiles on both the $y$ and $z$ axes 
$U_{o x} / U_{e}$ along the jet centreline for $N A R=0,2.5,7.5,12.5$ and 165 , respectively. Here, the result of the rectangular jet $(A R=10)$ by Sfeir [7] is plotted in the same figure for comparison. Each PC length for $N A R \geq 2.5$ is larger than that for $N A R=0$ (the rectangular jet) [8]. This reason is presumed as that there are the advection transport terms $\left(W \partial\left(U^{2} / 2\right) / \partial z\right.$ and $\left.V \partial\left(U^{2} / 2\right) / \partial y\right)$ of the mean flow kinetic energy by the inward secondary flow velocity on both the $z$ (the rectangular jet) and $y$ (the rectangular notch) axes as shown later in Figure 4, respectively. Furthermore, the length of the PC region $\left(U_{o x} / U_{e} \geq 1.0\right)$ varies with the value of $N A R$ and takes the largest value for $N A R=12.5$.

Figure 3 shows the streamwise variation of the longitudinal mean velocity profiles for all the $N A R$ cases on both the $y$ and $z$ axes, respectively. At first, the profiles on the $z$ axis at the section of $x / d=5$ for all the $N A R$ cases show potential core region $\left(U_{o x} / U_{e} \geq 1.0\right)$ and saddle-back shape in the region of $z / d<5.0$. At the section of $x / d=10$, the case of $N A R=0$ has only one peak at the jet end, on the other hand, all the cases for $N A R \geq 2.5$ have two peaks. The peak positions for all the $N A R$ cases at $x / d=10$ move toward the more inside region compared with those of the section of $x / d=5$, because the mean flow kinetic energy were transported toward the jet centre region by the inward advection transport term $\left(W \partial\left(U^{2} / 2\right) / \partial z\right)$ as mentioned above. In the region of $x / d \geq 20$, the peak positions for $N A R=0$ and 2.5 still move toward the jet centre region by the inward secondary flow
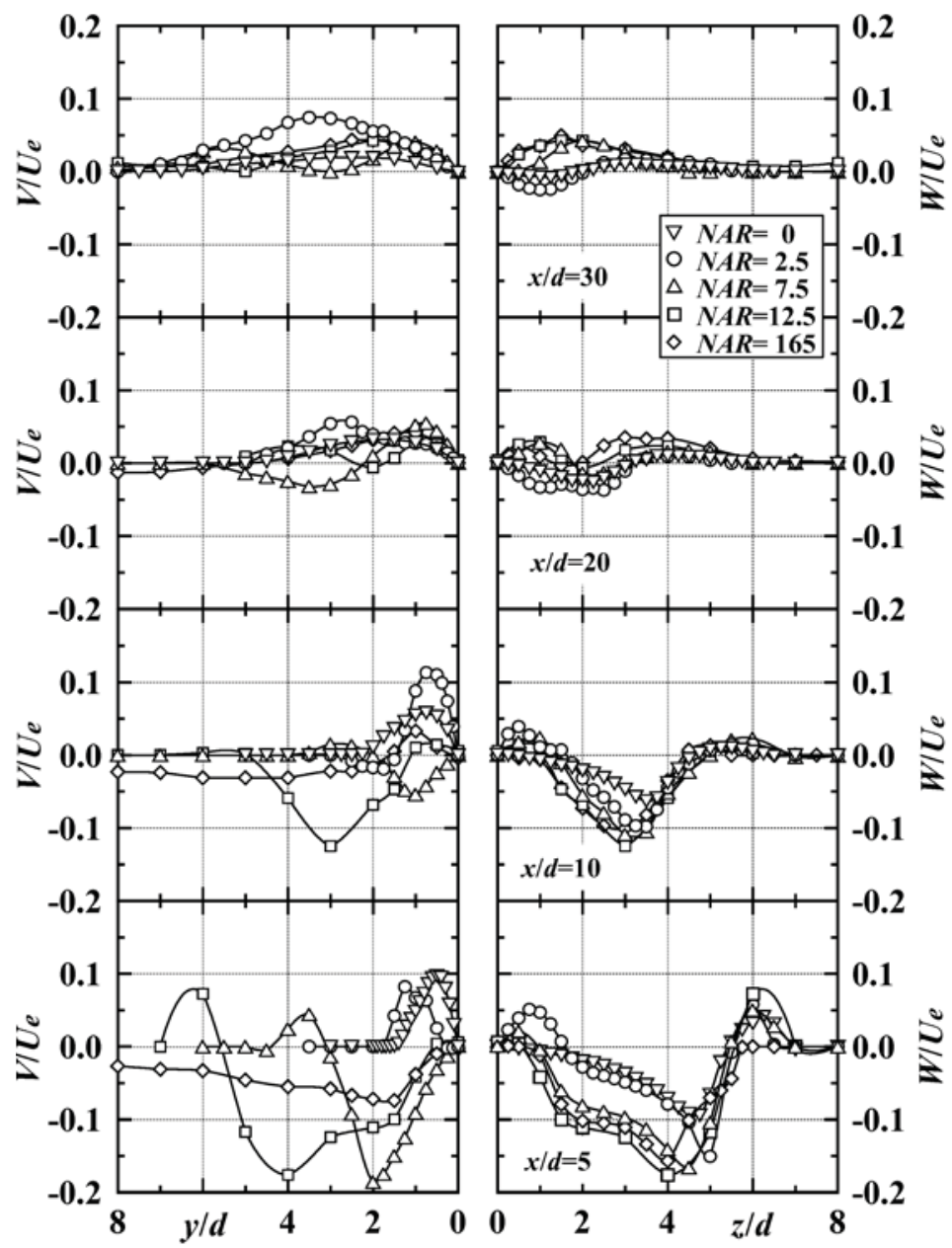

Figure 4: Secondary flow velocity profiles on both the $y$ and $z$ axes 
same as the result of $x / d=10$.

On the other hand, the profiles on the $y$ axis at the sections of $x / d=5$ for $N A R=2.5,7.5$ and 12.5 show each potential core region and saddle-back shape. However, the profile for $N A R=165$ takes almost the constant value on the $y$ axis. At the section of $x / d=10$, only two cases for $N A R=12.5$ and 165 show the saddle-back shape. Furthermore, in the region of $x / d \geq 20$, all profiles show monotonous decrease from the value of the jet centre $(y / d=0)$ to the notch jet end.

Figure 4 presents the profiles of the secondary flow velocity on both the $y$ and $z$ axes for all the NAR cases, respectively. At first, all the profiles on the $z$ axis at the section of $x / d=5$ have the inward secondary flow velocity. The magnitude of the inward secondary flow velocity for $N A R \geq 2.5$ is larger than that for $N A R=0$. At the sections of $x / d=10$ and 20 , there are also inward secondary flows for all the $N A R$ cases. All positions of the maximum value of the inward secondary flow velocity move toward the jet centre region. Finally at the section of $x / d=30$, the inward secondary flow velocities are found only for $N A R=0$ and 2.5 near the jet centre region.

Next, the profiles on the $y$ axis at the section of $x / d=5$ have the inward secondary flows for the cases of $N A R \geq 7.5$. The magnitude of the inward secondary flow velocity for $N A R=7.5$ and 12.5 takes large values. At the section of $x / d=10$, the maximum positions of the inward secondary flow velocity profiles for $N A R=7.5$ and 12.5 move toward the jet centre region. And at the section of $x / d=20$, there are still inward secondary flows for $N A R=7.5$ and 12.5 . Finally, at the section of $x / d=30$, there is no inward secondary flow in all the NAR cases.

From the results mentioned above, the magnitude and the direction of the inward secondary flow on both the $y$ and $z$ axes depend on the value of NAR. Using these features, it is presumed that the variation of $N A R$ can manipulate both the PC length and the development of the turbulent flowfield of the rectangular jet.

\subsection{TURBULENT VELOCITY SCALES}

In this chapter, the characteristics of the turbulent flowfield will be examined. Figure 5 shows the streamwise variation of turbulent velocity scales $q_{o x} / U_{e}$ along the jet centreline for all the $N A R$ cases. In the present experiment, the results in the upstream region will be focused, because it is presumed that the attachment of rectangular notch, which produces an inward secondary flow on the rectangular notch axis, makes a significant

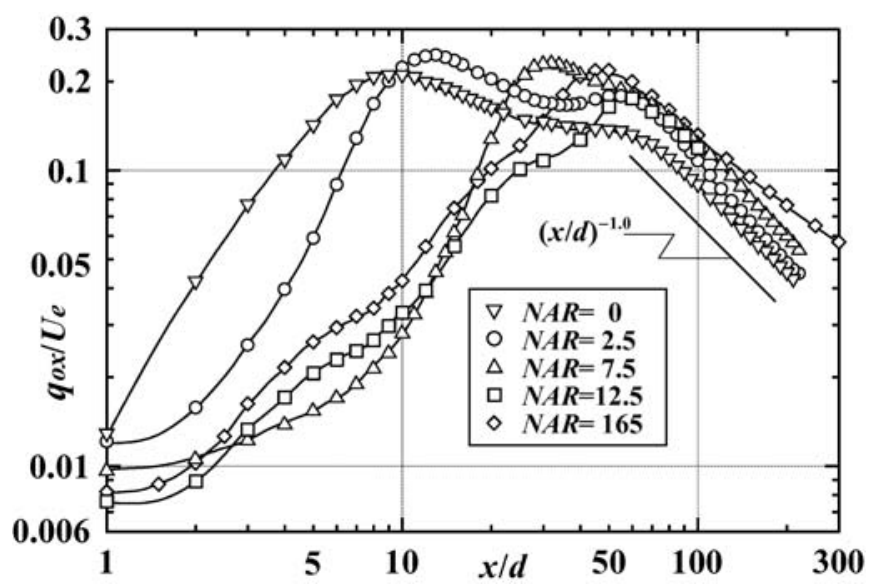

Figure 5: Turbulent velocity scales along the jet centreline 
contribution to the development of the present turbulent flowfield in the upstream region. At first, the magnitude of the value for $N A R=0$ is largest in the region until $x / d=9$. Then, in the region of $1 \leq x / d \leq 20$, the values of the velocity scales are suppressed for $N A R \geq 7.5$ compared with those for $N A R=0$ and 2.5.

From these results, it is found that the attachment of rectangular notch perpendicular to the rectangular nozzle suppresses the development of turbulent velocity scales near the jet centre in the upstream region.

\subsection{CONTOUR PLOTS OF TURBULENT KINETIC ENERGY}

Contour plots of the turbulent kinetic energy in the upstream region for all the NAR cases, will be examined to clarify the difference in the development of turbulent kinetic energy due to the effect of the inward secondary flow velocity on both the $y$ and $z$ axes. Figures 6 to 10 show the contour plots of turbulent kinetic energy $q_{u v} / U_{e}$ at two sections with a constant $x / d$ for all the $N A R$ cases, respectively.

The contour plot of $N A R=0$ at $x / d=5$ in Figure 6 shows the rectangular shape parallel to the rectangular nozzle axis and has the maximum value at $z / d \cong \pm 5.5$ on the $z$ axis. And the contour plot at the section of $x / d=30$ shows almost a circular shape and takes the maximum values at $z / d \cong 2.5$ on the $z$ axis. Comparing to that of $x / d=5$, it is presumed that the maximum positions on the $z$ axis are moved from $z / d \cong \pm 5.5$ to \pm 2.5 by the inward advection transport term $\left(W \partial\left(q^{2} / 2\right) / \partial z\right)$ of the turbulent kinetic energy due to the

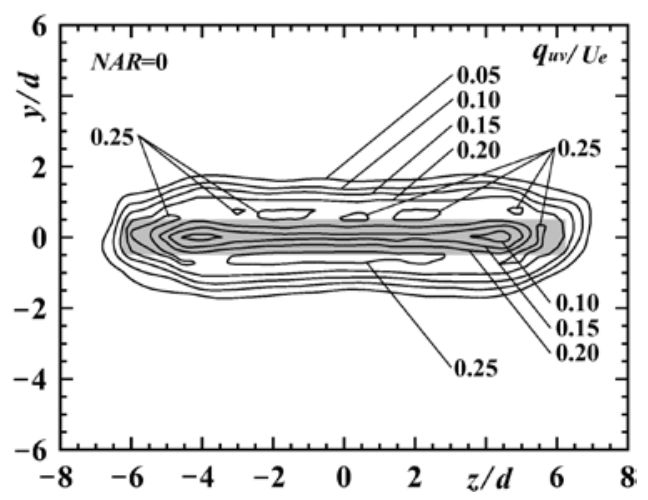

(a) $x / d=5$

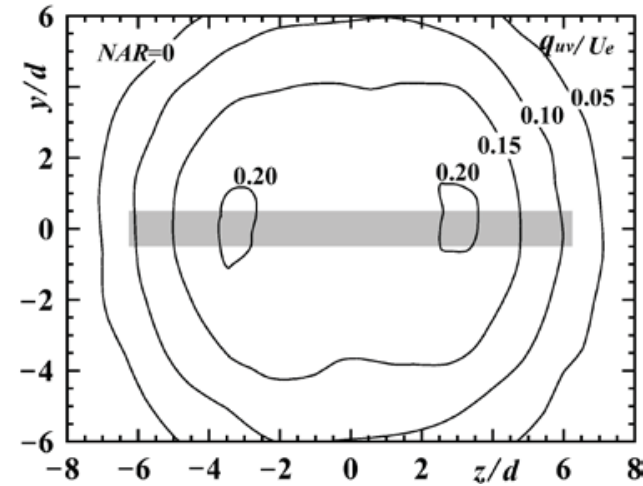

(b) $x / d=30$

Figure 6: Contour plots of turbulent kinetic energy $q_{u v} / U_{e}$ for $N A R=0$

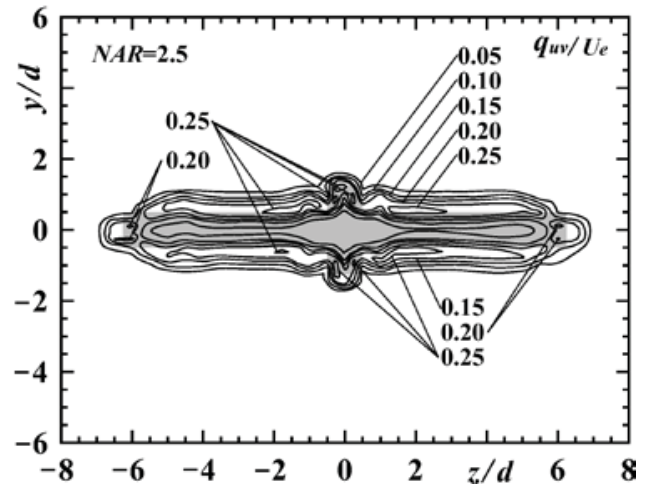

(a) $x / d=2$

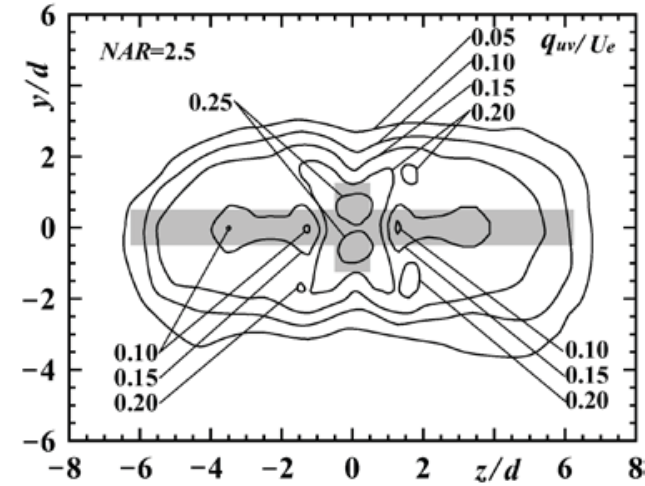

(b) $x / d=10$

Figure 7: Contour plots of turbulent kinetic energy $q_{u v} / U_{e}$ for $N A R=2.5$ 
inward secondary flow velocity as shown in Fig. 4.

Contour plot of $N A R=2.5$ at $x / d=2$ in Figure 7 shows almost a rectangular shape with small prominent regions on the $y$ axis and has the maximum value at $z / d \cong \pm 5.5$ on the $z$ axis. At the section of $x / d=10$, the contour plot takes the maximum value at $z / d \cong \pm 4.5$ on the $z$ axis because of the inward secondary flow velocity.

Next, the contour plot of $N A R=7.5$ at $x / d=5$ in Figure 8 shows a cruciform shape and

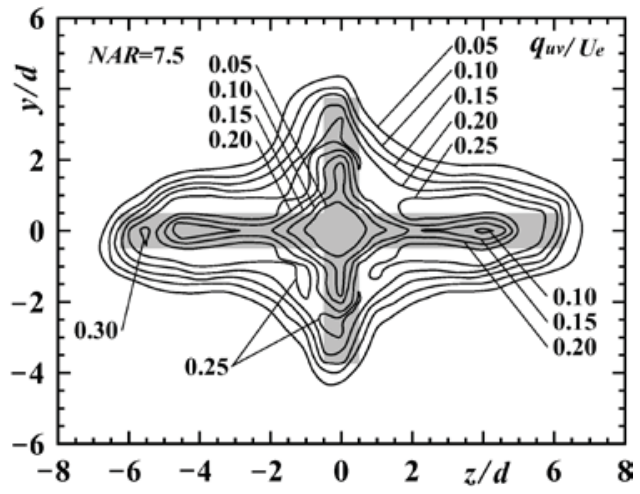

(a) $x / d=5$

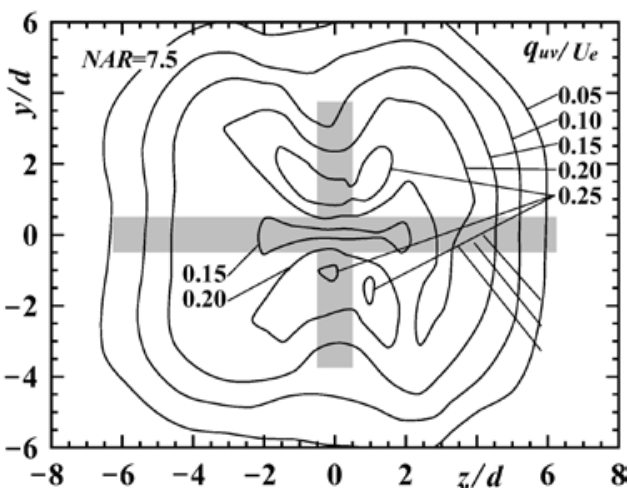

(b) $x / d=20$

Figure 8: Contour plots of turbulent kinetic energy $q_{u v} / U_{e}$ for $N A R=7.5$

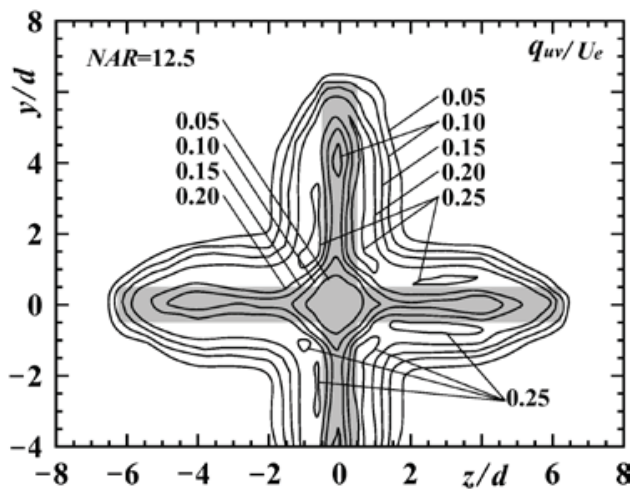

(a) $x / d=5$

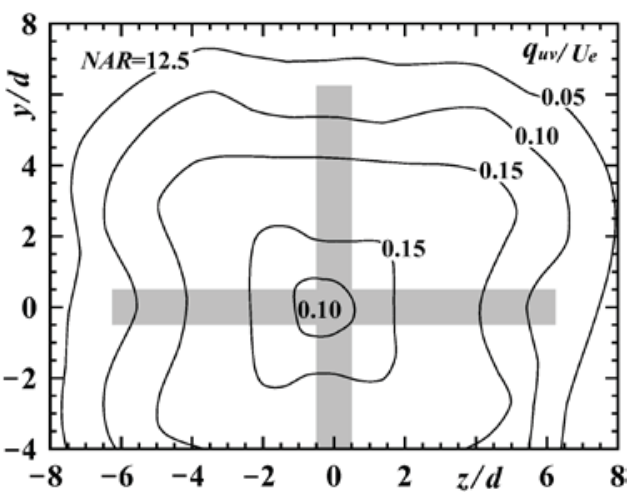

(b) $x / d=30$

Figure 9: Contour plots of turbulent kinetic energy $q_{u v} / U_{e}$ for $N A R=12.5$

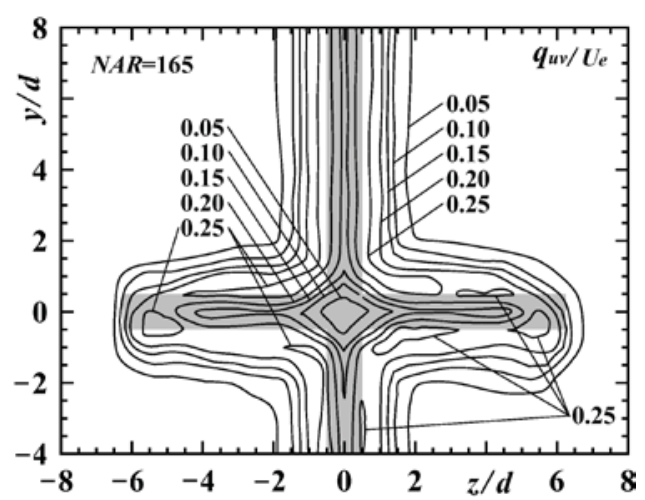

(a) $x / d=5$

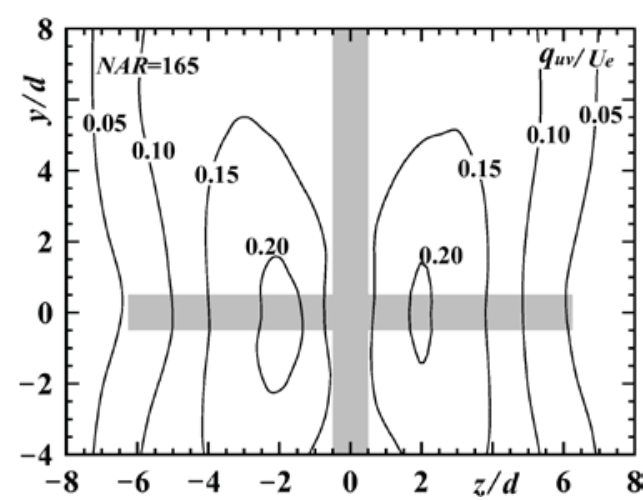

(b) $x / d=30$

Figure 10: Contour plots of turbulent kinetic energy $q_{u v} / U_{e}$ for $N A R=165$ 
takes the maximum values at $y / d \cong \pm 2.5$ on the $y$ axis and $z / d \cong \pm 5.5$ on the $z$ axis, respectively. The contour plot near the jet centre region shows a diamond-shaped region taking the local minimum value. This reason is explained as that the development of turbulent kinetic energy is suppressed by the inward advection transport which transports the mean flow kinetic energy with both high longitudinal mean velocity (see Fig.3) and low turbulent revel (see Figure 11 shown later) in the potential core region (see Fig.3) to the jet centre region on both the $y(y / d \leq 2.0)$ and $z(z / d \cong 4.5)$ axes at the section of $x / d=5$. Therefore, the value of the jet centre region for $N A R=7.5$ is kept smaller than that for $N A R=0$. At the section of $x / d=20$, the width of the contour plot on the $z$ axis is narrow compared with that of $x / d=5$ because of the same reason as the result on the $z$ axis for $N A R=2.5$.

The contour plot of $N A R=12.5$ at $x / d=5$ in Figure 9 shows a finite cruciform shape and takes the maximum value at $y / d \cong \pm 5.5$ and the minimum value at $y / d \cong \pm 4.0$ on the $y$ axis. It also takes the maximum and minimum values at the same locations on the $z$ axis. The contour plot near the jet centre region shows a diamond-shaped region taking the local minimum value. At the section of $x / d=30$, the contour plot shows the cruciform shape with long axes in the $y= \pm z$ directions, and takes the maximum value at $y / d \cong \pm 3.0$ and $z / d \cong \pm 3.0$ on the $y$ and $z$ axes, respectively. The movement of these maximum positions from $z / d \cong \pm 5.5$ to \pm 3.0 is caused by the advection transport of the turbulent kinetic energy by the inward secondary flow on both the $y$ and $z$ axes.

The contour plot shape along with the $z$ axis for $N A R=165$ at $x / d=5$ in Figure 10 shows the same tendency with another NAR cases. On the other hand, the contour plot along with the $y$ axis shows a shape of quasi two-dimensional jet. The contour plot in the jet centre region shows the diamond-shaped because of the same reason for the cases of $N A R=7.5$ and 12.5. At the section of $x / d=30$, the width of the contour plot on the $z$ axis is narrower than that of $x / d=5$. And the positions of the maximum value at $z / d \cong \pm 5.5$ of $x / d=5$ move to $z / d \cong \pm 2.0$.

From the results mentioned above, it is clarified that the development of the turbulent kinetic energy in the jet centre region for the cases of $N A R \geq 7.5$ is suppressed compared with the cases for $N A R=0$ and 2.5 due to the inward secondary flow velocity on both the $y$ and $z$ axes in the upstream region.

\subsection{TURBULENT KINETIC ENERGY PROFILES}

Figure 11 shows the streamwise variation of turbulent kinetic energy profiles on both the $y$ and $z$ axes for all the NAR cases, respectively.

All the profiles on the $z$ axis at $x / d=5$ show almost the same shape and take maximum values near the jet end $(z / d \cong \pm 5.5)$. The values of the jet centre region for $N A R \geq 2.5$ are smaller than that for $N A R=0$. This result coincides with that of the turbulent velocity scales in Fig.5. At the section of $x / d=10$, the values of $N A R \geq 7.5$ near the jet centre are suppressed compared with those for $N A R=0$ and 2.5. This result is explained as that fluid lumps having high longitudinal mean velocity and low turbulent revel in the potential core region for $N A R \geq 7.5$, are transported toward the jet centre region by the inward secondary flow on both the $y$ and $z$ axes as shown in Fig.4. Thus, it follows from what has been said that the attachment of the rectangular notch perpendicular to the rectangular nozzle suppresses the development of the turbulent kinetic energy near the jet centre in the upstream region, and the magnitude of the suppression effect depends on the value of $N A R$. At the section of $x / d=10$, the positions of the maximum value for all the $N A R$ 
cases move toward the more inside regions compared with those at the section of $x / d=5$ because of the inward secondary flow. Furthermore, at the sections of $x / d=20$ and 30, the positions of the maximum value for all the NAR cases moved toward the jet centre region until the section of $x / d=30$.

Next, the results on the $y$ axis will be examined. Each profile at $x / d=5$ except for $N A R=165$, takes the local minimum value near the jet centre and the maximum value near the region where the gradient of each longitudinal mean velocity profile shows the maximum value (see Fig.3). The profile of $N A R=165$ has almost the constant value in the region of $y / d \geq 2.0$. At the section of $x / d=10$, the magnitude of the value near the jet centre for $N A R \geq 7.5$ is suppressed compared with those for $N A R=0$ and 2.5, this is the same reason as the result on the $z$ axis. Therefore, the magnitude of the value near the jet centre for $N A R \geq 7.5$ is kept small. Furthermore, the positions of the maximum value move toward the jet centre region except for $N A R=0$. At the section of $x / d=20$, the positions of the maximum value for $N A R=7.5$ and 12.5 still move toward the jet centre region. The magnitude of the value at the jet centre $(y / d=0)$ for $N A R \geq 7.5$ approaches to that for $N A R=0$. At the section of $x / d=30$, the positions of the maximum value move toward the outside except for $N A R=7.5$.

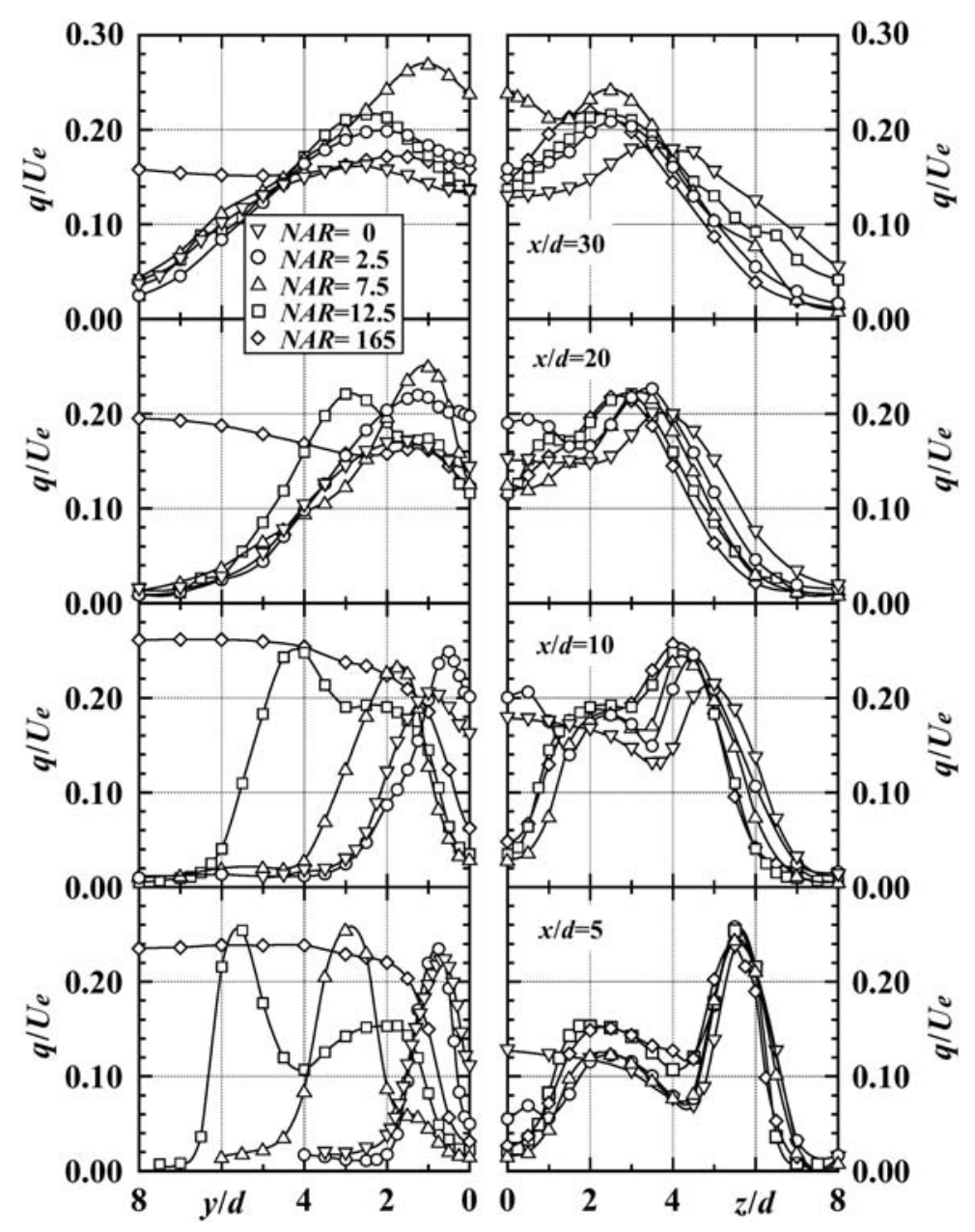

Figure 11: Turbulent kinetic energy profiles on both the $y$ and $z$ axes 


\section{Conclusions}

From the present experiment in the turbulent flowfield of the turbulent air jet issuing from rectangular nozzle $(A R=12.5)$ with a rectangular notch $(N A R=2.5,7.5,12.5$ and 165 ) at the midspan, the main conclusions of the present study can be summarized as follows:

(1) The magnitude and the direction of the inward secondary flow velocity on both the rectangular nozzle and rectangular notch depend on the value of NAR. The variation of NAR manipulates both the PC length and the development of the turbulent flowfield of the rectangular jet.

(2) The attachment of rectangular notch perpendicular to the rectangular nozzle suppresses the development of turbulent velocity scales near the jet centre in the upstream region, and the magnitude of the suppression effect depends on the value of NAR.

(3) The development of the turbulent kinetic energy in the jet centre region for the cases of $N A R \geq 7.5$ is suppressed compared with the cases of $N A R=0$ and 2.5 due to the inward secondary flow velocity on both the $y$ and $z$ axes in the upstream region.

\section{REFERENCES}

[1] Trentacoste, N. and Sforza, P.M., Further experimental results for threedimensional free jets. AIAA Journal, 5(5), 1967, pp.885-891.

[2] Sforza, P.M. and Stasi, W., Heated three-dimensional turbulent jets. Transaction of the ASME, Journal of Heat Transfer, 101(2), 1979, pp.353-358.

[3] Marsters, G.F. and Fotheringham, J., The influence of aspect ratio on incompressible, turbulent flows from rectangular slots. Aeronautical Quarterly, 31(4), 1980, pp.285-305.

[4] Fujita, S. and Osaka, H., Three-dimensional jets issuing from cruciform nozzles having four aspect ratios (1st Report. Mean Flow Properties). Transactions of the Japan Society of Mechanical Engineers, Series B, 53(488), 1987, pp.1142-1149.

[5] Fujita, S. et al., Turbulent jet issuing from a quasi two-dimensional nozzle with a rectangular notch at the midspan (streamwise variation of mean velocity field). Transactions of the Japan Society of Mechanical Engineers, Series B, 65(631), 1999, pp.905-911.

[6] Quinn, W.R and Marsters, G.F., Upstream influence on turbulent jet flows from cruciform nozzles. Aeronautical Journal, 89(882), 1985, pp.55-58.

[7] Sfeir, A.A., Investigation of three-dimensional turbulent rectangular jets. AIAA Journal, 17(10), 1979, pp.1055-1060.

[8] Fujita, S. et al., Turbulent jets issuing from the rectangular nozzle with a rectangular notch at the midspan, Fluid Structure Interaction $V$, Proceedings of Fifth International Conference on Fluid Structure Interaction, WIT Press, 2009, pp.61-70. 